\title{
MAPPING FOREST FIRE IMPACT FROM LANDSAT-TM IMAGERY
}

\author{
Agustín Lobo ${ }^{a}$, Nicolau Pineda ${ }^{a}$, Rafael Navarro-Cedillo ${ }^{b}$, Pilar Fernandez-Rebollo ${ }^{b}$, Francisco J. \\ Salas $^{\mathrm{b}}$, José-Luis Fernández-Turiel $^{\mathrm{a}}$ and Arturo Fernández-Palacios ${ }^{\mathrm{c}}$
}

\author{
anstituto de Ciencias de la Tierra "Jaume Almera" (CSIC), Lluis Solé Sabarís s/n 08028 Barcelona, \\ Spain \\ ${ }^{b}$ E.T.S.I. Agrónomos y Montes, Universidad de Cordoba, Av. Menéndez Pidal s/n, Apdo. 3048, 14080 \\ Córdoba, Spain \\ 'Servicio de Evaluación de Recursos Naturales, Consejería de Medio Ambiente, \\ Junta de Andalucía, Cartuja 93, 41071 Sevilla, Spain.
}

\begin{abstract}
We address the problem of estimating fire impact in Mediterranean forests based on Landsat-TM imagery, for which we have used a forest fire in Andalucia (S. Spain) as a case of study. We processed two LANDSAT-TM scenes, acquired before and after the date of the fire. The post-fire scene was segmented and a table of segment statistics was submitted to a hierarchical model-based agglomerative clustering. The boundary defined by the classification closely matches the boundary defined by helicopter. We also produced an image classification of the pre-fire image based on image segmentation and canonical analysis and studied the trajectories of burnt and not-burnt centroids in the Kauth-Thomas plane. The trajectories indicated the existence of a differential response to fire and to phenologic change. We modeled the post-fire conditions as if the region had not been burnt and defined an Index of Fire Impact as the difference between the actual and the modeled second Kauth-Thomas component. The Index of Fire Impact is significantly related to field estimates, but with an scatter that introduces uncertainity in the inversion.
\end{abstract}

Keywords: Fire, environmental impact, forest, image segmentation.

\section{INTRODUCTION}

Fire is a typical disturbance in Mediterranean landscapes and is a major concern for forest managers in the region. Remotely-sensed imagery has proven to be an important tool for detecting forest fire occurrence and for studying the joint dynamics of fire and vegetation at a range of scales. At a local scale, detailed information on land cover is necessary not only to understand this joint dynamics, as well as for the assessment of risk and impact, including economic evaluations. The detailed study of some important land cover characteristics requires the analysis of imagery with high spatial resolution. These characteristics include different vegetation properties, like classes based on the relative

For further information

A.L. (correspondence): email: alobo@ija.csic.es; http://pangea.ija.csic.es/alobo

Telephone: 34 933302716; Fax: 34934110012 
abundance of dominant species, biomass, the relative abundance of "green" and "not-green" biomass or the abundance of pyrophyle plants, as well as more transient properties like water content. Some of these variables can be derived from the imagery through appropriate physical models and some others can be inferred by combining field and image data within a geostatistical approach.

Our goal is to use remotely-sensed imagery to improve estimates of fire impact in Mediterranean forests based on Landsat-TM imagery, for which we have used a forest fire in Andalucia ( $S$. Spain) as a case of study. Our project is intended to produce a precise definition of the boundary of the fire scar and geographically-explicit information on pre-fire conditions and fire response. This information is critical for a forest management aimed to reduce fire risk and facilitate post-fire recover.

\section{AREA OF STUDY}

We studied a forest fire that occurred on May 30th, 1995 in the area of Monte El Madronalejo $\left(37^{\circ} 38^{\prime}\right.$ N. $6^{\circ} 26^{\prime} \mathrm{W}$ ). Climate is Mediterranean with potential evapotranspiration higher than precipitation from May to October. Vegetation is dominated by Mediterranean evergreen shrub land. Plantations of pines have a significant cover. most often intermingled due to the recovery of native species.

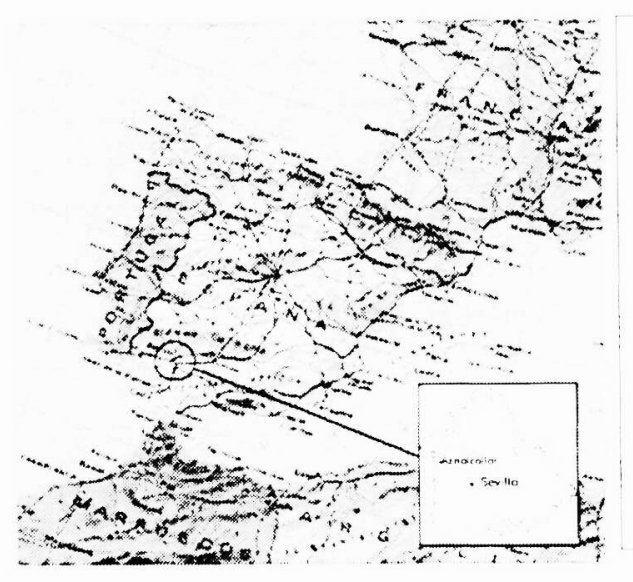

(a)

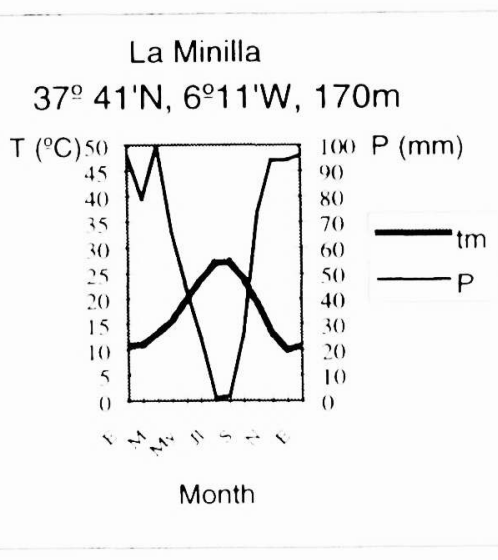

(b)

Figure 1. (a) Situation of the area of study; (b) monthly mean temperature ( $t m$ ) and precipitation $(P)$ in the station that is closest to the area of study (La Minilla).

\section{MATERIALS AND METHODS}

\subsection{Detection of the fire scar}

We used a pre-fire LANDSAT-TM image acquired on June $5^{\text {th }}$, 1995 . After a standard georectification by means of a second-order polynomial (mean square error $<30 \mathrm{~m}$ ), we submitted a subscene to the Iterative Mutually-Optimum Region Merging (IMORM ${ }^{1}$ ). After an edge preserving ${ }^{2}$. IMORM divides 
the image into facets and merges those facets that are mutually most-similar under the constrain of a user-defined threshold.

We calculated the Principal Components (PC) transform and built a multi-variate table with the average values of the first three PC per segment. We run a model-based hierarchical agglomerative clustering ${ }^{3}$ with the multi-variate table (Fig. 2a). A plot of the Bayes factor indicated that the number of classes that maximizes the likelihood of the partition given the data was 10 (Fig. 2b). A 10-classes classification was produced by cutting the dendrogram at the corresponding dissimilarity level. The classified image was produced by re-labeling segments to their class.

A helicopter established the boundary of the fire scar by over-flying the perimeter at the moment of fire extinction and recording the coordinates with its GPS navigator. This data were used to generate the helicopter estimate of a "burnt" - "not-burnt" partition of the region. The helicopter and the image classification were compared in a contingency table. According to this table, the image was reclassified to "burnt" and "not-burnt" categories.

\subsection{Pre-fire conditions.}

We applied methods based on image segmentation, hierarchical clustering and discriminant analysis to a pre-fire Landsat-TM image of 20-7-1994. The image was first segmented with IMORM, analogously to the preceding section, but with a more strict threshold, which rendered an image divided in more numerous and smaller segments. A sample of 3000 segments was submitted to a model-based hierarchical clustering and used the resulting 16-classes classification to define a linear canonical transform of the TM sub-scene. We run a second hierarchical clustering in the canonical space, which produced a 10-classes classification. The centroids and covariance matrices for the classes were calculated and all segments were classified by maximum likelihood.

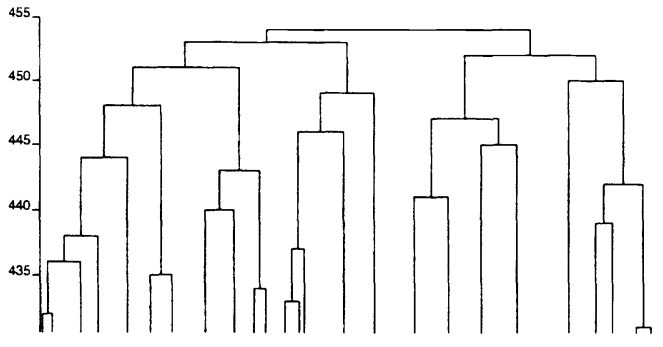

(a)

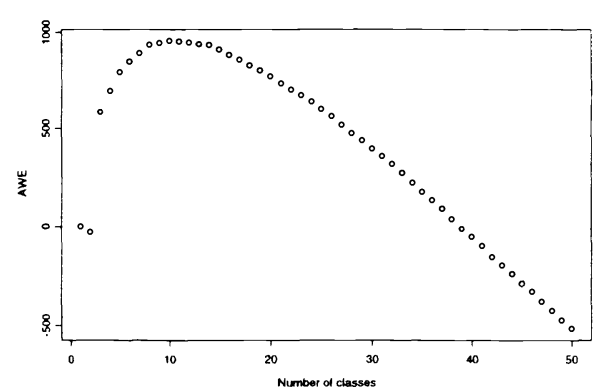

(b)

Figure 2. Hierarchical agglomerative clustering of the multivariate table produced from the $99 \%$ segmentation and the Principal Component image. (a) Dendrogram; (b) Plot of the Bayes factor. 


\subsection{Pre-fire to post-fire trajectories}

We first rendered the pre- and post-fire images spectrally comparable. A formal atmospheric correction would have required reliable measurements of atmospheric optics or, at least, vertical profiles of temperature (radio-soundings) from which to estimate the optical properties of the atmosphere through an appropriate model.

As neither measurements were available for any date, we standardized both atmospheric conditions by linear regression. A close-to-linear relationship between at-sensor reflectance of the same TM band at different dates is to be expected if images for both dates are acquired under clear-sky conditions ${ }^{4}$, which is a normal requirement for purchasing Landsat-TM imagery.

We transformed both images to at-sensor reflectance, calculated a robust linear regression by LeastTrimmed Squares regression ${ }^{5}$ between the not-burnt region in both images and applied the regression to the pre-fire image.

We conducted an stratified exploratory analysis of the change between the pre- and the post-fire image. We used the classification that has been described in the preceding section and studied the trajectories in the Kauth-Thomas space, for which we draw segments from the pre-fire to the post-fire positions of the centroids.

\subsection{Index of fire impact}

We have defined an Index of Fire Impact (IFI) by comparing the actual decrease of the value in the second Kauth-Thomas component (TC-2) to an empirically modeled value that simulates the scene as if fire had not occurred. The model uses the classification of the pre-fire image and calculates the median values of the Kauth-Thomas components in the not-burnt part of the post-fire image. Each pixel in the modeled image takes then the value of the median of its class. The index of fire impact was defined as the difference between the actual post-fire TC- 2 and the modeled TC- 2

The values of the IFI were compared to field-assessed Degrees of Fire Impact in 158 sites $^{6}$ by means of box plots.

\section{RESULTS}

We represented the post-fire classification geographically with the helicopter-defined fire scar boundary overlaid (Fig. 3). Three classes (7,8 and 9) presented an almost perfect match with the burnt area and were labeled as "burnt", leaving the rest as "not-burnt". The boundary defined by both categories closely matches the boundary defined by the helicopter. The total area affected by fire as estimated by this classification is $2,553.8$ ha.

We produced a summary interpretation of the pre-fire classification by means of plots of the values of the classified sub-sample of segments in the canonical and Kauth-Thomas spaces ${ }^{7}$, and the inspection of high-resolution digitized color aerial photography (Fig. 4). 


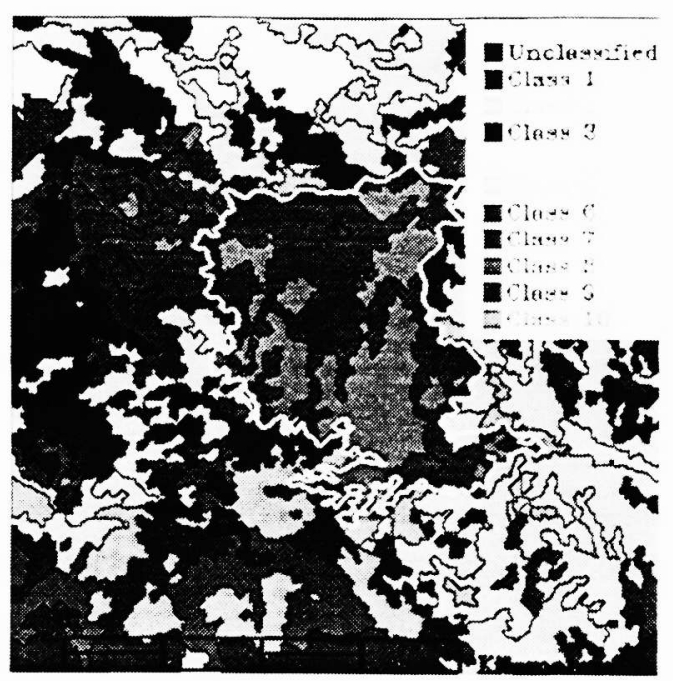

(a)

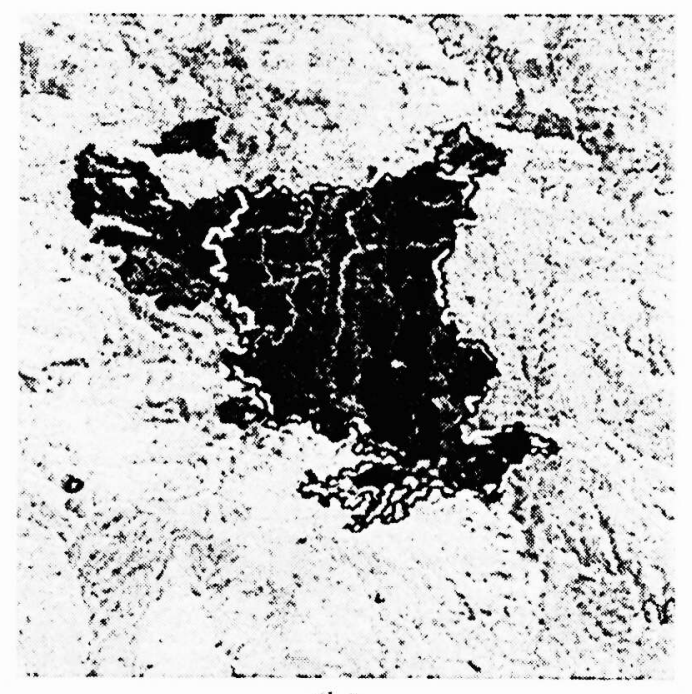

(b)

Figure 3. (a) Ten-classes hierarchical classification of the segmented image with the helicopter-defined fire boundary overlaid as a white vector; (b) The color composite ( $b / w$ reproduction) defined by the first three Kauth-Thomas components with the two fire boundaries overlaid: white vector. helicopter-defined; black vector, reclassification result.

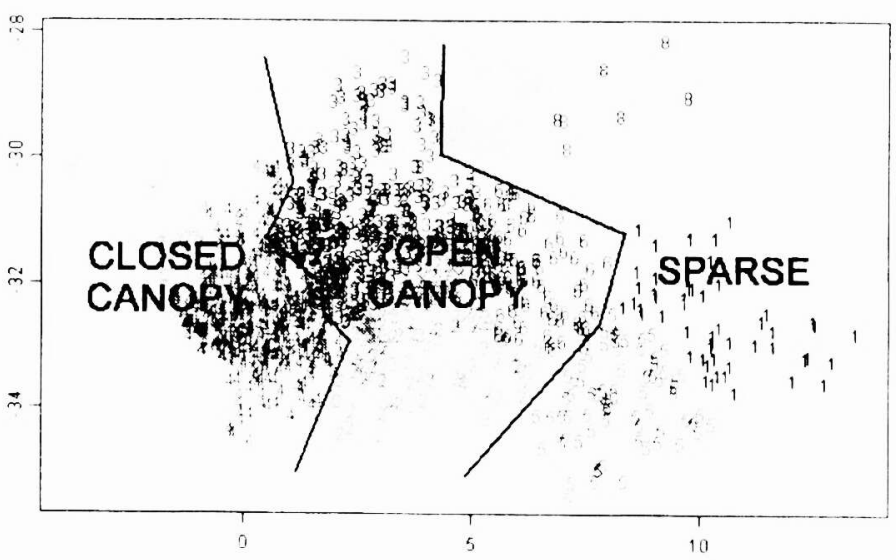

Figure 4. Plot of the sampled segments in the canonical space. Numbers refer to the final 10 classes.

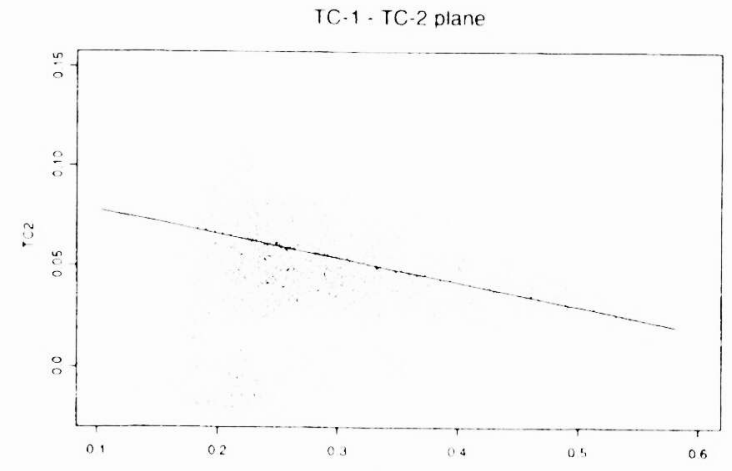

Figure 5. Scatter plot in the TC-1 - TC-2 plane of pre-fire (green) values and post-fire (red) pixel values. The line of the robust (least-trimmed) regression for the pre-fire image is also shown. 
A plot of a random sample of 1000 pixels of pre-fire and post-fire values in the space defined by the first two Kauth-Thomas components shows that the burnt pixels clearly clustered in the lower-left part of the plot (Fig. 5).

Trajectories from the pre-fire to the post-fire positions of the centroids (Fig. 6) are consistent with the nature of the different land cover categories.. Arrows pointing to open circles describe the change of the not burnt surface between pre- and post-fire dates. Land cover dominated by bare soil (classes 1, 8 and 10) decreases in brightness but increases in greeness and wetness. On the other extreme, closed forest (classes 4, 7 and 9) increases in brightness while decrease greeness and, slightly, wetness. Open forest classes (2, 3 and 6$)$ decreases in all three components. These dynamics imply that vegetation was drier at the post-fire date, while differences in bare soil reflectance are probably due to the change in solar incidence angle.

Arrows pointing to solid circles describe change due to fire. Classes 1, 8, 10 and 5 (dominated by bare soil) did not get burnt, and actually acted as barriers to fire propagation. Closed canopy forest (classes 4,7 , and 9) severely decreased greeness and wetness with slight or no change in brightness, while open canopy forest (classes 2, 3 and 6) slightly decreased brightness in adition to a severe decrease in greeness. The decrease in brightness was more severe as the percent of openess was higher, as in class 6. These responses indicate that green vegetation lost the near-infrared - red contrast and that short, dry understorey became darker. Class 6, the most open of the three open canopy forest classes, had a different response to fire in terms of wetness: while all other classes slightly or moderately decreased wetness, wetness increased in burnt surfaces classified as 6 .
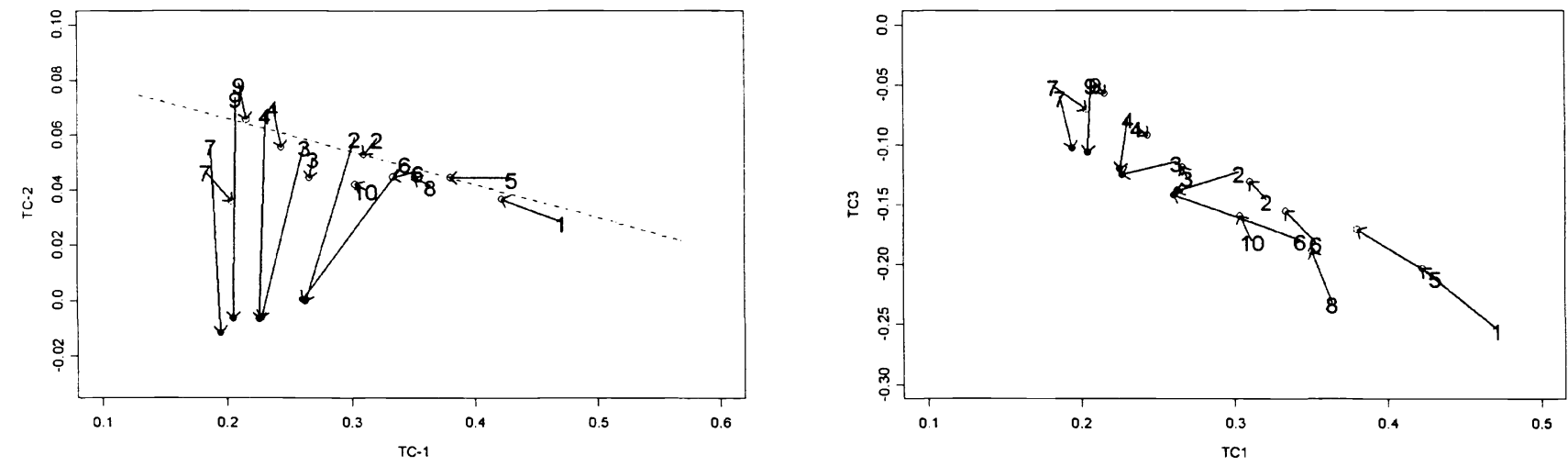

Figure 6. Trajectories in the Kauth-Thomas space. Numbers represent median values for the 10 classes. Arrows point from values in the pre-fire TM image to the post-fire TM image. Open circles represent median values of the not-burnt part of the post-fire image, while solid circles represent the median values of the burnt part

The Index of Fire Impact calculated as the difference between the actual and modeled TC-2 shows a significant relationship to the field-assessed Degree of Fire Impact, but there is a considerable overlap, in particular between consecutive degrees (Fig. 7). 


\section{Post-fire TC-2 - Model Medians}

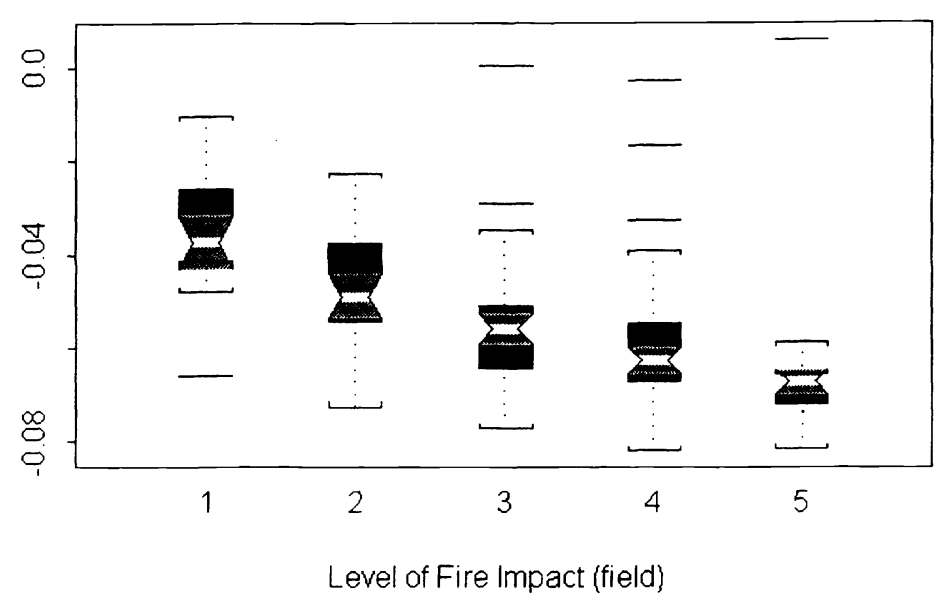

Figure 7. Box plots of the values of the index of fire impact (y-axes) stratified by the field-observed levels of fire impact (X-axes). White bands indicate the medians; square brackets, the range; notches, $95 \%$ confidence intervals of the medians.

\section{DISCUSSION AND CONCLUSIONS}

Although fire has a notorious effect on surface reflectance and fire scars use to be prominent features in the TM imagery, the assessment of the degree of fire impact is more subtle and requires a detailed analysis to consider the differential response of landcover categories. Also, it is desirable that the assessment of fire impact involve the comparison of pre- and post-fire images, which, due to acquisition, atmospheric and/or economic reasons, often are separated by a significant length of time. Such a time lag may involve some phenological change, which typically is dependent on the vegetation type also. Therefore, both differential response to fire and differences in phenological change among landcover types, along with the subtlety of the variable of interest, advise the use of detailed pre-fire vegetation maps.

The simulation of the not-burnt post-fire reflectance (or image transformations, like the Kauth-Thomas) is an adequate method to include differences in the per-class response to fire and in the per-class phenologic response. This is proven by the significant relationship of the Index of Fire Impact to the field assessments, but the overlap between the distributions introduces uncertainity in the inversion. A future challenge is, precisely, narrowing the range and/or studying the spatial distribution of the error. Nevertheless, it must be noted that the mentioned uncertainity does not come only from the side of the analysis of the remotely-sensed imagery: some inconsistency on the estimation of Degrees of Fire Impact in the field as well as location errors are likely to be present.

Finally, we can summarize our conclusions as follows:

A process based on image segmentation is very efficient at detecting the boundary of the fire scar as well as at producing a land cover map of pre-fire conditions. Classes so produced show a consistent response to both phenologic and fire-induced change. 
An estimation of fire impact cannot be done from a simple comparison of pre-fire and post-fire imagery if, as in our case, there is a significant time span between the dates of both images.

(iii) An Index of Fire Impact defined as the difference between the actual second KauthThomas component and the modeled value is significantly related to the field-estimated Degree of Fire Impact, although the overlap between distributions introduces uncertainity in the inversion.

\section{ACKNOWLEDGEMENTS}

We thank the Servicio de Evaluación de Recursos Naturales of the Consejería de Medio Ambiente of the Junta de Andalucia (Spain), for providing imagery and valuable information, as well as Jesús SanMiguel Ayanz and Francisco Rodríguez-Silva for their friendly support and valuable comments. This work has been funded by the Space Applications Institute of the Joint Research Center of the European Union in Ispra (Italy).

\section{REFERENCES}

1. A. Lobo, "Image segmentation and discriminant analysis for the identification of landscape units in Ecology", IEEE Transactions on Geoscience and Remote Sensing 35, pp. 1136-1145, 1997.

2. M. Nagao and T. Matsuyama, A Structural Analysis of Complex Aerial Photographs, Plenum, New York, 1980.

3. J.D. Banfield and A.E. Raftey, "Model-based Gaussian and non-Gaussian Clustering", Biometrics, 49, pp. 803-821, 1992.

4. V. Caselles and M.J. López-García, "An alternative simple approach to estimate atmospheric correction in multitemporal studies", International Journal of Remote Sensing 10, pp. 11271134, 1989.

5. P.J. Rousseeuw, "Least median of squares regression", Journal of the American Statistical Association 79, pp. 871-888, 1984

6. R.M. Navarro, P. Fernández., F.J. Salas and C. Navarro, "Evaluación de daños producidos por un incendio y regeneración posterior de la vegetación. Aplicación de imágenes Landsat-TM a su caracterización y seguimiento", ETSIAM Report (Universidad de Córdoba) for the Consejería de Medio Ambiente (Junta de Andalucía), 1997.

7. E.P. Crist. and R.C: Cicone, "A physically based transformation of Thematic Mapper data: the TM Tasseled Cap", IEEE Transactions on Geoscience and Remote Sensing 22, pp. 256-263, 1984. 